PROCEEDINGS OF THE

AMERICAN MATHEMATICAL SOCIETY

Volume 127, Number 12, Pages 3733-3737

S 0002-9939(99)04902-3

Article electronically published on May 10, 1999

\title{
CONSONANCE AND TOPOLOGICAL COMPLETENESS IN ANALYTIC SPACES
}

\author{
AHMED BOUZIAD
}

(Communicated by Alan Dow)

\begin{abstract}
We give a set-valued criterion for a topological space $X$ to be consonant, i.e. the upper Kuratowski topology on the family of all closed subsets of $X$ coincides with the co-compact topology. This characterization of consonance is then used to show that the statement "every analytic metrizable consonant space is complete" is independent of the usual axioms of set theory. This answers a question by Nogura and Shakhmatov. It is also proved that continuous open surjections defined on a consonant space are compact covering.
\end{abstract}

A topological space $X$ is said to be consonant if the co-compact topology on the set of all closed subsets of $X$ coincides with the upper Kuratowski topology. The class of consonant spaces was introduced by Dolecki, Greco and Lechicki in [2], [3] and was recently studied rather intensively. It was noticed by Nogura and Shakhmatov in [8] that two other classical topologies (automatically) coincide on the set of closed subsets of a consonant space, namely, the Fell topology and the Kuratowski topology. In [3], among other things, it is proved that every Cechcomplete space is consonant. It is also known that metrizable consonant spaces are hereditarily Baire [1] (i.e. every closed subspace of a metrizable consonant metric space is a Baire space). These results prompted Nogura and Shakhmatov in [8, Problem 11.4] to ask whether consonant metrizable spaces are (Čech-)complete. This remained one of the central open problems in the theory of consonance. For coanalytic metrizable spaces, this problem is entirely solved: A co-analytic metrizable space $X$ is consonant if and only if $X$ is Polish (see [1]). In this note we show that the statement "every analytic metrizable consonant space is complete" is independent of the usual axioms of set theory (Theorem 6). It is also proved that continuous open surjections defined on a consonant space are compact covering (Corollary 8), which gives a generalization of the classical theorem of Pasynkov stated for Cechcomplete spaces. Corollary 8, along with a construction of Michael [6], allows us to give a new and topological proof of the nonconsonance of the usual space of rationals.

This note is based on a characterization of consonance in terms of a special property of lower semicontinuous set-valued maps (Theorem 2). Concerning topological completeness, an important and recent result of this kind, achieved by van Mill,

Received by the editors October 7, 1996 and, in revised form, February 10, 1998.

1991 Mathematics Subject Classification. Primary 54A35; Secondary 54B20, 54C60.

Key words and phrases. Upper Kuratowski convergence, co-compact topology, analytic spaces, consonant spaces. 
Pelant and Pol [7], says that complete metrizable spaces are those metric spaces satisfying the compact-valued selection theorem of Michael.

Recall that a multifunction (or a set-valued map) $\varphi$ defined on a space $Y$ which takes its values in the set $2^{X}$ of nonempty closed subsets of the space $X$ is said to be lower semicontinuous (or briefly l.s.c.) if, for every open set $U \subset X$, the set $\{y \in Y: \varphi(y) \cap U \neq \emptyset\}$ is open in $Y$. A subset $A$ of $X$ which meets all values of $\varphi$ will be called a section of $\varphi$.

As in [3], for every collection $\mathcal{D}$ of subsets of $X$ we denote by $\mathcal{O}(\mathcal{D})$ the set of open subsets of $X$ which contain some member of $\mathcal{D}$. A collection $\mathcal{H}$ of open sets in $X$ is said to be compact if $\mathcal{H}=\mathcal{O}(\mathcal{H})$ and for each collection $\mathcal{U}$ of open sets in $X$ such that $\bigcup \mathcal{U} \in \mathcal{H}$ there is a finite subcollection $\mathcal{V} \subset \mathcal{U}$ such that $\bigcup \mathcal{V} \in \mathcal{H}$. A first example of a compact collection of open sets is given by $\mathcal{O}(\mathcal{K})$ for every family $\mathcal{K}$ of compact subsets of $X$.

Definition 1. A space $X$ is said to be consonant if, for every compact collection of open sets $\mathcal{H}$ in $X$, there is a family $\mathcal{K}$ of compact sets in $X$ such that $\mathcal{O}(\mathcal{K})=\mathcal{H}$.

Of course this definition is not the original one [3]; it corresponds to the characterization of consonance given in [3, Theorem 3.3]. We also inform the reader that, as in [3], no separation axioms are a priori assumed, consequently compact subsets are not assumed to be closed.

The following is the criterion of consonance mentioned above.

Theorem 2. A space $X$ is consonant if and only if, for every l.s.c. multifunction $\varphi: Y \rightarrow 2^{X}$, where $Y$ is compact, every open section of $\varphi$ contains a compact section of $\varphi$.

Proof. Let us show that the condition is necessary. Suppose that $X$ is consonant and let $\varphi: Y \rightarrow 2^{X}$ be a l.s.c. multifunction, where $Y$ is a compact space. Denote by $\mathcal{H}$ the collection of all open sections of $\varphi$. It follows from the compactness of $Y$ and the fact that $\varphi$ is l.s.c. that $\mathcal{H}$ is a compact collection. Let $U \in \mathcal{H}$ and let $K \subset U$ be a compact set such that $V \in \mathcal{H}$ for every open set $V \subset X$ which contains $K$. Let $y \in Y$. Clearly $X \backslash \varphi(y)$ is not an open section of $\varphi$, i.e. $X \backslash \varphi(y) \notin \mathcal{H}$. In particular, $K \not \subset X \backslash \varphi(y)$ and thus $\varphi(y) \cap K \neq \emptyset$. We conclude that $K$ is a compact section of $\varphi$.

Conversely, suppose that the condition of the theorem is satisfied, and let $\mathcal{H}$ be a nonvoid compact collection of open sets in $X$. Without loss of generality we may assume that $\emptyset \notin \mathcal{H}$. Consider the set $Y$ of all open sets $U \subset X$ such that $U \notin \mathcal{H}$. Let $\varphi: Y \rightarrow 2^{X}$ be the multifunction defined by $\varphi(U)=X \backslash U$, for each $U \in Y$. We endow $Y$ with the coarser topology making the multifunction $\varphi$ l.s.c.

Let us show that $Y$ is compact. Let $\mathcal{U}$ be a collection of open sets in $X$ such that $\varphi(y) \cap \bigcup \mathcal{U} \neq \emptyset$ for each $y \in Y$. To prove that $Y$ is compact it suffices to show, by the Alexander subbase theorem, that there is a finite subcollection $\mathcal{V} \subset \mathcal{U}$ such that $\varphi(y) \cap \bigcup \mathcal{V} \neq \emptyset$ for every $y \in Y$. We have $\bigcup \mathcal{U} \in \mathcal{H}$; indeed, otherwise we obtain $\bigcup \mathcal{U} \in Y$ and then $(X \backslash \bigcup \mathcal{U}) \cap \bigcup \mathcal{U} \neq \emptyset$, which is impossible. Consequently, by the compactness of $\mathcal{H}$, there is a finite sub-collection $\mathcal{V} \subset \mathcal{U}$ such that $\bigcup \mathcal{V} \in \mathcal{H}$. Let $U \in Y$ and let us verify that $\varphi(U) \cap \bigcup \mathcal{V} \neq \emptyset$. Suppose that this is not the case. Then we have $\bigcup \mathcal{V} \subset U$, which is impossible because $U \notin \mathcal{H}$. Hence $Y$ is compact.

Now, let $O \in \mathcal{H}$; since $O$ is an open section of $\varphi$, there is a compact set $K \subset O$ which meets every value of $\varphi$; and, for each open set $U \subset X$ such that $K \subset U$, we have $U \in \mathcal{H}$. This proves that $X$ is consonant. 
For a space $X$ we denote by $\mathcal{K}(X)$ the family of non-empty compact subsets of $X$. We endow $\mathcal{K}(X)$ with the upper Vietoris topology, that is the topology for which the sets $U^{+}=\{K \in \mathcal{K}(X): K \subset U\}$, with $U$ an arbitrary open subset of $X$, form a base. For a l.s.c. set-valued map $\psi: Y \rightarrow 2^{X}$ let $\mathcal{S}(\psi)$ be the (possibly empty) set of all compact sections of $\psi$. We consider $\mathcal{S}(\psi)$ as a subspace of $\mathcal{K}(X)$ with the induced topology.

Recall that a space $X$ is said to be a Baire space if every countable intersection of dense open subsets of $X$ is a dense subset of $X$.

Lemma 3. Let $X$ be a Polish space and $\psi: Y \rightarrow 2^{X}$ a l.s.c. set-valued map defined on a compact space $Y$. Then $\mathcal{S}(\psi)$ is a non-empty secound countable Baire space.

Proof. It is proved in [3] that Čch-complete spaces are consonant. Since Polish spaces are Cech-complete, it follows from Theorem 2 that $\mathcal{S}(\psi)$ is non-empty. The second countability of $\mathcal{S}(\psi)$ follows immediately from that of $X$. To show that $\mathcal{S}(\psi)$ is a Baire space, fix a complete metric $d$ compatible with the topology of $X$ and let $\left(\mathcal{O}_{n}\right)_{n \in \omega}$ be a decreasing sequence of dense open subsets of $\mathcal{S}(\psi)$. Let $U$ be an open subset of $X$ such that $\mathcal{S}(\psi) \cap U^{+} \neq \emptyset$. Choose by induction a sequence $\left(\left(U_{0}^{n}, \ldots, U_{k_{n}}^{n}\right)\right)_{n \in \omega}$ of finite sequences of open subsets of $X$, the diameter of each $U_{i}^{n}$ being less than $1 /(n+1)$, such that

$$
\emptyset \neq \mathcal{S}(\psi) \cap U^{+} \cap\left(U_{0}^{n} \cup \ldots \cup U_{k_{n}}^{n}\right)^{+} \subset \mathcal{O}_{n},
$$

and such that the closure (in $X$ ) of each $U \cap U_{i}^{n+1}$ is contained in some $U \cap U_{j}^{n}$. This construction guarantees that the set $K=\bigcap_{n \in \omega} U \cap\left(U_{0}^{n} \cup \cdots \cup U_{k_{n}}^{n}\right)$ is a non-empty compact subset of $X$ for which the sequence $\left(U \cap\left(U_{0}^{n} \cup \cdots \cup U_{k_{n}}^{n}\right)\right)_{n \in \omega}$ forms a base of neighbourhoods. Let $y \in Y$ and suppose that $K \cap \psi(y)=\emptyset$. Then there is some $n \in \omega$ such that $U \cap\left(U_{0}^{n} \cup \cdots \cup U_{k_{n}}^{n}\right) \cap \psi(y)=\emptyset$, which is impossible because of $(*)$. Hence $K$ is in $\mathcal{S}(\psi)$. This proves that $\bigcap \mathcal{O}_{n}$ is dense in $\mathcal{S}(\psi)$.

For a set-valued map $\psi: Y \rightarrow \mathcal{P}(X)$, where $\mathcal{P}(X)$ denotes the set of all subsets of the space $X$, we use $\bar{\psi}$ to denote the set-valued map defined by $\bar{\psi}(y)=\overline{\psi(y)}$ for each $y \in Y$.

Lemma 4. Let $X$ be a consonant space, $F$ a proper closed subset of $X$ and $\psi$ : $Y \rightarrow 2^{X \backslash F}$ a l.s.c. set-valued map. Then the set $\mathcal{S}_{F}(\bar{\psi})=\{K \in \mathcal{S}(\bar{\psi}): K \cap F=\emptyset\}$ is open, non-empty and dense in $\mathcal{S}(\bar{\psi})$.

Proof. Note that the set-valued map $\bar{\psi}: Y \rightarrow 2^{X}$ is well defined and l.s.c.. It is also clear that the set $\mathcal{S}_{F}(\bar{\psi})$ is an open subset of $\mathcal{S}(\bar{\psi})$. Let us show that $\mathcal{S}_{F}(\bar{\psi})$ is a dense subset of $\mathcal{S}(\bar{\psi})$ (hence non-empty, by Lemma 3). Let $U \subset X$ be an open subset of $X$ such that $\mathcal{S}(\bar{\psi}) \cap U^{+} \neq \emptyset$ and let $K_{0} \in \mathcal{S}(\bar{\psi})$ be such that $K_{0} \subset U$. For each $y \in Y$ we have $\emptyset \neq \bar{\psi}(y) \cap K_{0} \subset \overline{\psi(y)} \cap U$, hence $\emptyset \neq \psi(y) \cap U=\psi(y) \cap(U \backslash F)$. Therefore, the multifunction $\gamma: Y \rightarrow 2^{U \backslash F}$ defined by $\gamma(y)=\overline{\psi(y)} \cap(U \backslash F)$ is well defined and l.s.c.. Since $U \backslash F$ is an open subspace of the consonant space $X$, it is consonant [3]; hence, by Theorem 2, there is a compact section $K$ of $\gamma$. It is clear that $K \in U^{+} \cap \mathcal{S}_{F}(\bar{\psi})$.

In order to use Theorem 2 to establish that a regular space $X$ is consonant it is sufficient to show that every l.s.c. set-valued map, which takes its values in $2^{X}$ and is defined on a compact space, has a compact section. Indeed, let $\psi: Y \rightarrow 2^{X}$ be 
such a map and $U \subset X$ an open section of $\psi$. Then, by the regularity of $X$, there is an open section $V \subset X$ of $\psi$ such that $\bar{V} \subset U$. Now, for any compact section $K$ of the l.s.c. set-valued map $y \in Y \rightarrow \overline{\psi(y) \cap V} \in 2^{X}$, the compact set $K \cap \bar{V}$ is a compact section of $\psi$ contained in $U$.

The following assertion was inspired by [7, Theorem 5.1]

Theorem 5 (MA). Let $X$ be a Polish space. For any collection $\mathcal{F} \subset 2^{X}$ of cardinality less than $\mathfrak{c}$, the cardinality of the continuum, the subspace $Z=X \backslash \cup \mathcal{F}$ of $X$ is consonant.

Proof. Let $\psi: Y \rightarrow 2^{X \backslash \cup \mathcal{F}}$ be a l.s.c. set-valued map, where $Y$ is a compact space. Since the space $X \backslash \bigcup \mathcal{F}$ is regular, by the remark above, it suffices to show that $\psi$ has a compact section. As in Lemma 4 , for every $F \in \mathcal{F}$ denote by $\mathcal{S}_{F}(\bar{\psi})$ the set $\{K \in \mathcal{S}(\bar{\psi}): K \cap F=\emptyset\}$. From Lemmas 3 and 4 and the well known fact that Martin's Axiom (MA) implies that in a second countable Baire space the intersection of less than $\mathfrak{c}$ open dense subsets is dense (see, for example, [10, Theorem 14, p. 497]), it follows that $\bigcap_{F \in \mathcal{F}} \mathcal{S}_{F}(\bar{\psi}) \neq \emptyset$, which implies that $\psi$ has a compact section.

Recall that a metrizable space is called analytic if it is a continuous image of a Polish space.

Theorem 6. The statement "all analytic metric consonant spaces are completely metrizable" is independent of the usual axioms of set theory.

Proof. The arguments are the same as those in [7, Remark 5.2]. Let $C$ denote the Cantor set. By Martin and Solovay [5, 3.1 and 3.2] it is consistent with MA $+\neg \mathrm{CH}$ that for every $A \subset C$ with cardinality $\aleph_{1}$, the space $C \backslash A$, which is consonant by Theorem 5 but not Čech-complete by the Cantor-Bendixon Theorem, is analytic.

On the other hand, there is a model of set theory in which every analytic metrizable space which is not completely metrizable contains a closed homeomorphic copy of the rationals $\mathbb{Q}$ (see Kanover and Ostrovskir [4]). Since $\mathbb{Q}$ is not consonant [1] (see also Remark 9 below) and consonance is hereditary with respect to closed subsets, in this model every analytic metrizable consonant space is complete.

It is shown in [3, Proposition 7.3] that consonance is not hereditary with respect to $G_{\delta}$-subsets. However, Lemma 4 implies the following:

Proposition 7. If $X$ is a regular consonant space such that the set of its compact subsets endowed with the upper topology is hereditarily Baire, then all $G_{\delta}$ subsets of $X$ are consonant.

A classical theorem of Pasynkov states that every continuous open surjection defined on a Cech-complete space is compact covering [9]. Recall that a continuous map $f$ from a space $X$ onto a space $Y$ is called compact covering if for every compact set $A \subset Y$ there is a compact set $B \subset X$ such that $f(B)=A$. The next statement extends Pasynkov's result to the class of consonant spaces.

Corollary 8. Every open continuous onto map $f: X \rightarrow Y$, where $X$ is a consonant space and $Y$ is a Hausdorff space, is compact covering.

Proof. Let $K_{0}$ be a compact subset of $Y$. We must show that there is a compact set $K_{1} \subset X$ such that $f\left(K_{1}\right)=K_{0}$. The openness of $f$ implies that the multifunction 
which sends each $y \in K_{0}$ to $f^{-1}(y)$ in $2^{X}$ is l.s.c.. It follows from Theorem 2 that there is a compact set $K \subset X$ such that $f^{-1}(y) \cap K \neq \emptyset$ for each $y \in K_{0}$. Put $K_{1}=K \cap f^{-1}\left(K_{0}\right)$.

Remark 9. To conclude this note, let us mention that Corollary 8 can be used to give a topological proof of the nonconsonance of the usual set $\mathbb{Q}$ of rationals. Answering a question from [2], the nonconsonance of $\mathbb{Q}$ was established in [1] by using a measure theoretical argument. The proof is as follows: Let $C$ denote the Cantor set and, following [6], let $A \subset C \times C$ be such that the projection map $\pi: A \rightarrow C$ is open, onto and not compact covering. The subspace $A$ of $C \times C$ is therefore not consonant (Corollary 8). In [11], Saint Raymond constructs a set $A$ satisfying these conditions and, in addition, is such that there exists a perfect onto mapping $f: A \rightarrow \mathbb{Q}$. It follows from $[8$, Theorem 8.1] that $\mathbb{Q}$ is not consonant.

\section{ACKNOWLEDGEMENT}

The author is indebted to the referee for suggesting several improvements in the presentation of this note.

\section{REFERENCES}

[1] Bouziad A., Borel measures in consonant spaces, Top. Appl. 70 (1996), 125-132. MR 97c:54010

[2] Dolecki S., G.H. Greco and A. Lechicki, Sur la topologie de la convergence supérieure de Kuratowski, C. R. Acad. Sci. Paris 312 (1991), 923-926. MR 92c:54007

[3] Dolecki S., G.H. Greco and A. Lechicki, When do the upper Kuratowski topology (homeomorphically, Scott topology) and the co-compact topology coincide?, Trans. Amer. Math. Soc. 8 (1995), 2869-2884. MR 96c:54010

[4] Kanoveǐ V.G. and A.V. Ostrovskiǐ, On non-Borel FII-sets, Soviet Math. Dokl. 24 (1981), 386-389.

[5] Martin D.A. and R.M. Solovay, Internal Cohen extensions, Ann. Math. Logic 2 (1970), 143178. MR 42:5787

[6] Michael E., A theorem on semi-continuous set-valued functions, Duke Math. Jour. 26 (1959), 647-651. MR 22:229

[7] van Mill J., J. Pelant and R. Pol, Selections that characterize topological completeness, Fund. Math., vol. 149, 1996, pp. 127-141. MR 97b:54027

[8] Nogura T. and D. Shakhmatov, When does the Fell topology on a hyperspace of closed sets coincide with the meet of the upper Kuratowski and the lower Vietoris topologies?, Top. Appl. 70 (1996), 213-243. MR 97f:54011

[9] Pasynkov B.A., On open mappings, Dokl. Akad. Nauk SSSR 175 (1967), 292-295 (in Russian); English transl.: Soviet Math. Dokl. 8 (1967), 853-856. MR 36:862

[10] Rudin M.E., Martin's Axiom, in: J. Barwise, ed., Handbook of Mathematical Logic, NorthHolland Publishing, 1977, pp. 491-501. MR 56:15351

[11] Saint Raymond J., Caractérisation d'espaces Polonais, Sém. Choquet (Initiation Anal.) 5 (1971-1973), 10 p.. MR 57:12811

Département de Mathématiques, Université de Rouen, CNRS UPRES-A 6085, 76821 Mont Saint-Aignan, France

E-mail address: Ahmed.Bouziad@univ-rouen.fr 